\title{
Alternative futures for the DSM revision process: iteration $V$. paradigm shift
}

Kenneth S. Kendler and Michael B. First

\section{Summary}

Two major approaches can be used for the up-coming revisions of DSM-V and ICD-10: an 'iterative model' in which incremental changes are made or a 'paradigm shift model' in which the existing approach is jettisoned in favour of a new nosological model. We explore each of these two approaches and conclude that although they both have strengths and limitations, our field is not currently ready for a paradigm shift.

\section{Declaration of interest}

M.B.F. consults with pharmaceutical companies to provide diagnostic training for clinical trials. In the past 12 months, he has consulted with Cephalon, GlaxoSmithkline, Memory Pharmaceuticals, Worldwide Clinical Trials and i3 research.
The two major psychiatric classification systems, the American Psychiatric Association's DSM-IV ${ }^{1}$ and the World Health Organization's ICD- $10,{ }^{2}$ are being revised for the first time since the 1990s. Both researchers and clinicians have expressed frustration with the approach taken by DSM-IV and ICD-10 where disorders are made up of diagnostic categories defined descriptively in terms of symptoms that have been observed to covary in individuals, supplemented by optional severity dimensions (mild, moderate and severe) and one cross-cutting dimension for assessment of functioning (i.e. the Global Assessment of Functioning on Axis V). In adopting this approach, DSM-III ${ }^{3}$ assumed that, as in general medicine, the phenomenon of symptom covariation was an indication that their presentation could be explained by a common underlying aetiology and pathophysiology and that, over time, these aetiological factors would be elucidated. Unfortunately, in the 30 years that has elapsed since the publication of DSM-III, the goal of clarifying this pathophysiology has remained elusive. Despite the discovery of many initially promising candidates over the years, no laboratory marker has been shown to be diagnostically useful for making any DSM diagnosis. ${ }^{4,5}$ Other evidence suggesting that the current classification lacks validity include: high rates of diagnostic comorbidity; lack of treatment specificity for the diagnostic categories; evidence that distinct syndromes share a genetic basis; and the high rates of individuals requiring the use of the diagnostically unspecific not otherwise specified (NOS) category. Some investigators have also raised concerns that continued use of the current diagnostic paradigm might impede future research efforts. ${ }^{6}$

There are two different methods for revising our psychiatric nosology: the 'iterative model', in which incremental changes are made while retaining the fundamental assumptions of the existing model; or the 'paradigm shift model', where the underlying paradigm is discarded in favour of a fundamentally new approach. We explore these two approaches, provide some examples of how each model might work and conclude by noting that despite the well-recognised limitations with our current paradigm, our field is not yet ready for an entirely new paradigm.

\section{The iterative model}

The iterative model assumes that using increasingly rigorous empirical methods, each subsequent revision of our diagnostic system will produce improvements over its predecessor. ${ }^{7}$ Over time, this process will slowly move our nosology from the rough constructs we now call 'disorders' towards a better and better approximation of the 'true' psychiatric diseases as they exist in nature. The iterative model is evolutionary and cumulative in nature. The architects of DSM-III- ${ }^{8}$ and DSM-IV implicitly utilised this approach. For example, the introduction of DSM-III-R begins with:

'This is the revision of the third edition of the APA'S DSM, better known as DSM-III-R. The last sentence of the introduction to DSM-III, published in 1980, stated "DSM-III is only one still frame in the ongoing process of attempting to better understand mental disorders." DSM-III-R represents another still frame.' (p. xvii).

The DSM-IV revision strategy, which called for making changes only if there was sufficient evidence to justify such a change, also implicitly followed this iterative model.

The main advantage of the iterative model is its conservativeness. It limits the rate of nosological change, allowing each transition, from one iteration of the classification to the next, to be straightforward thereby minimising disruption to clinical practice and research. Furthermore, if each iteration of the nosology succeeds in more closely approximating the true nature of psychiatric disorders, this model would produce a steady improvement in the validity of the diagnostic system.

The iterative model has several potential problems, however. Most importantly, it assumes that the paradigm on which the classification is based is correct. Regarding the DSM paradigm, these include the assumptions that: mental disorders can be conceptualised as syndromes of covarying symptoms; classification should be descriptive rather than aetiological; and splitting categories into small putatively more homogeneous entities is the correct approach despite the consequent flourishing of comorbidity.

Moreover, the iterative model assumes continuity over time in our approach towards validating psychiatric disorders. The model works only if the methods used to determine validity remain consistent. What happens if dramatic technical breakthroughs in genetics, imaging or neuroscience cast the problems of psychiatric nosology in an entirely new light? The application of such new methods to our nosology would likely disrupt the smooth evolutionary approach of the iterative model.

\section{The paradigm shift model}

At some point, the limits of the current paradigm may be reached and a general consensus will arise that the system is broken. Rather than fixing the old model, the best approach might then be to discard it and start over. Thomas Kuhn suggested that scientific fields go through long periods of 'normal science' (e.g. our 
iterative model), working out details of results within a dominant paradigm. ${ }^{9}$ Eventually, anomalies and problems with that paradigm accumulate to a point where a scientific revolution is sparked. The old paradigm is replaced with a new one.

Dissatisfaction with the current DSM approach to classification has led to a call, from some quarters, for a paradigm shift in psychiatric classification. What might a 'post-revolutionary' DSM or ICD look like? There are a wide range of possibilities that we can only hope to briefly sketch in this article. One approach would retain the categorical approach to diagnosis but completely replace the diagnostic categories, based on clinical tradition, with empirically derived prototypes. An alternative approach would be to adopt a 'bottom-up' empirical methodology for creating categories. This process could seek to develop a 'theory-free' set of input variables including not only symptoms and signs but also other potential variables such as genetics, biological measures, brain imaging, treatment response and course of illness. These variables would then be collected on a large sample of individuals and the results handed to a team of statisticians who, in consultation with experts in nosology, would isolate the optimal number of categories of illness necessary to explain the pattern of results.

Another paradigm-shifting approach would be to reject diagnostic categories altogether and replace them with dimensions. Such dimensions could be selected in a top-down manner in which a single set of all-encompassing dimensions are chosen from a number of competing possibilities, or using a bottom-up approach in which new dimensions would emerge empirically from data-sets combining the various elements described in the first approach.

However, the paradigm shift that has been most frequently advocated would be to replace the current descriptive classification with one that is aetiologically based. ${ }^{4,10,11}$ Despite the plethora of research advances that support psychiatry's view of mental illness as neurobiologically based diseases, not a single DSM category is defined in terms of brain processes. Proponents of an aetiologically based DSM blame the field's inability to define disorders aetiologically on current technological and methodological limitations, and assume that it is only a matter of time until advances allow for the arrival of the necessary major neurobiological breakthroughs. Only then, based on clear molecular and/or neurobiological mechanisms, can we develop a 'real' aetiologically based nosological system that will justly take its place alongside the diagnostic systems of the rest of medicine.

There are several ways in which the vision of an aetiologically based approach to psychiatric disorders might arise. First, it might prove possible to subdivide our broad syndromes into more aetiological homogeneous subgroups, each with a relatively clear single cause. Many biomedical syndromes, which initially appeared to be singular illnesses, are now understood as syndromal presentations with many distinct aetiological causes. Mental retardation (intellectual disability) was long considered a single syndrome but is now known to have a multitude of distinct environmental, chromosomal and genetic aetiologies. Could the major psychiatric syndromes eventually be split into a variety of specific and aetiologically homogeneous entities?

A critical area of research that raises serious questions about the viability of this 'decomposition' of our broad syndromes into more aetiologically homogenous diseases is psychiatric genetics. Although the evidence for aggregate genetic effects for the major psychiatric syndromes is very strong, 20 years of work in linkage and candidate gene and, now, in genome-wide association studies, has produced strong evidence against the hypothesis that subforms of these disorders are the result of genes of large effect. ${ }^{12,13}$ Perhaps new discoveries in neuroscience or other areas of research not yet conceptualised will yield powerful single causes for many of our current syndromes. Our sense - only an 'educated guess' - is that this is unlikely because most psychiatric disorders are inherently multifactorial. Not only are they influenced by diverse sets of risk factors, but these factors are bundled together to produce many different assemblages of jointly sufficient factors.

Another vision of an aetiologically based system would rely on 'emergent simplification'. That is, at the most basic level, a wide diversity of individual molecular changes can increase risk for particular psychiatric syndromes. However, by moving 'up' towards the levels of cognitive and affective neuroscience, then we may be able to define a single common pathophysiological pathway. If we could clarify and measure that process, then perhaps a robust one-to-one relationship would emerge that would ground our syndromes solidly in neuroscience. ${ }^{10}$

We do not know enough to judge the plausibility of this argument. However, there is reason for scepticism. Central nervous system lesions (e.g. stroke or tumour) can produce quite variable psychiatric symptoms. One pathological process, infection of the central nervous system with the spirochaete Treponema pallidum, can present with a primary psychotic, manic, depressive or demented clinical picture. Huntington's disease presents in some individuals with psychotic symptoms but in most with only movement symptoms and eventually dementia.

The complexity of the human central nervous system and the variation in its structure and function, which results from genetic diversity and environmental exposures during development, may mean that the relationship between basic biological processes and psychiatric symptoms and signs is much more frequently many-to-many than one-to-one.

Finally, our aetiological model for psychiatric illness may be fundamentally incorrect. Like biological species, psychiatric disorders may not arise from single 'essences', but rather result from a stable network of causes that interact across levels. ${ }^{14}$ This approach may provide the most realistic avenue for psychiatry to ground our diagnoses in aetiology by expanding our aetiological concepts from solitary causes to disordered systems.

\section{Continuity $v$. change?}

At what point does it make sense to abandon one paradigm in favour of another? Ideally, the shift should be organic, occurring at a point at which the advantages of the new paradigm become so overwhelming that to continue with the existing paradigm would make no sense. But what happens if a shift is driven by a new paradigm whose advantages over the existing paradigm are tentative, more theoretical than practical, appealing but not 'road tested'? Might we be at risk of making a shift because 'the grass looks greener' with the new paradigm? Adopting a new paradigm before its time runs the risk of forcing the field to radically alter its diagnostic practices only to end up disillusioned once the serious flaws of the paradigm shift become apparent. Critically, a successful revolution requires not only wide-spread dissatisfaction with the old paradigm, but the emergence of a compelling new one that addresses successfully its predecessor's limitations.

The emergence of DSM-III from DSM-II ${ }^{15}$ has many of the features of a paradigm shift. DSM-I ${ }^{16}$ and DSM-II had been roundly criticised for being unreliable and tied to unproven psychodynamic aetiological theories. Meanwhile, the introduction of descriptive operationalised diagnostic criteria in the 1970s (e.g. the Feighner, ${ }^{17}$ and Research Diagnostic Criteria) ${ }^{18}$ transformed psychiatric research by allowing researchers around the world to utilise the same reliable diagnostic definitions. The revolution embodied by DSM-III involved advocating the adoption of a paradigm that had already been widely embraced by the research community for general clinical and administrative use. 


\section{Implications for DSM-V and ICD-11}

As discussed, the DSM nosology underwent a major paradigm shift with the release of DSM-III and has, since that time, roughly followed an iterative model. As dictated by the iterative approach, the architects of each subsequent edition intended them to reflect an incremental improvement over its predecessor in terms of reliability and validity.

What is in store for DSM-V and ICD-11? Dissatisfaction with the current DSM-IV descriptive approach has resulted in aspirations for a paradigm shift for DSM-V. One of the stated goals of the DSM-V Research Agenda was 'to transcend the limitations of the current DSM paradigm and to encourage a research agenda that goes beyond our current ways of thinking' (p.xix), with the ultimate goal of adopting an 'etiologically and pathophysiologically-based diagnostic system' (p. 35). ${ }^{19}$

There are clearly important limitations to the current DSMbased paradigm and many legitimate reasons for wanting to discard the current descriptive categorical paradigm in favour of something more valid. However, successful paradigm shifts need more than that. Changing paradigms will place a considerable burden on the DSM user-community in terms of the costs of learning the new system, implementing new diagnostic and assessment procedures and creating a significant discontinuity in diagnostic data-sets. To sustain a scientific revolution, both the push of increasing dissatisfaction with the old paradigm and the pull of a new paradigm that can definitively address many of these concerns are needed. Although our field clearly has the 'push' for change, we do not yet have a strong enough 'pull' from a superior alternative paradigm that will successfully address our concerns.

\section{Conclusion}

Revisions of psychiatric classifications can follow one of two approaches: an iterative approach in which incremental changes are made to the existing paradigm, or else a paradigm shift in which the existing paradigm is jettisoned in favour of a new approach. Despite the serious shortcomings of the current descriptive categorical paradigm adopted by DSM-IV and ICD-10, the current evidence suggests that both DSM-V and ICD-11 are likely to continue this approach and adopt an iterative approach to revision. As noted by Regier and colleagues, 'the major difference between DSM-IV and DSM-V will be the more prominent use of dimensional measures in DSM-V' (p. 649). ${ }^{20}$ Since the proposed severity and cross-cutting dimensions are essentially an expansion of dimensional aspects of the current DSM paradigm (e.g. severity specifiers, Global Assessment of Functioning scale), these changes fall comfortably within the current paradigm.

The fact that the DSM revisions have traditionally occurred at regular intervals, rather than when the field is ripe for change, is at least partly responsible for the dilemma of deciding whether to shift paradigms for a particular revision or having the paradigm shift arise more organically. There is, however, one future anticipated change in the DSM revision process that might prove beneficial to future psychiatrists entrusted with making these decisions. The American Psychiatric Association has indicated its intention to change its revision model so that sections of the classification can be revised on an as-needed basis, driven by the presence of compelling empirical evidence indicating the need for change. ${ }^{20}$ Given that successful scientific revolutions are hard to accomplish on a predetermined schedule, this development might facilitate a more natural evolution to a new diagnostic paradigm.
Kenneth S. Kendler, MD, The Virginia Institute of Psychiatric and Behavioral Genetics and Departments of Psychiatry and Human and Molecular Genetics, Medical College of Virginia/Virginia Commonwealth University, Virginia; Michael B. First MD, Biometrics Research Department, New York State Psychiatric Institute and Department of Psychiatry, Columbia University, College of Physicians and Surgeons, New York, USA

Correspondence: Dr Kenneth S. Kendler, Virginia Institute for Psychiatric and Behavioral Genetics, Virginia Commonwealth University Medical School, Box 980126, 800 E. Leigh Street, Room 1-123, Richmond, VA 23298-0126, USA. Email: kendler@hsc.vcu.edu

First received 17 Dec 2009, final revision 29 Apr 2010, accepted 19 May 2010

\section{Acknowledgements}

Peter Zachar, PhD, Kenneth Schaffner, MD, PhD, Robert Krueger, PhD, Jerome Wakefield, $\mathrm{PhD}, \mathrm{DSW}$, and Daniel Pine, MD, provided helpful comments on earlier versions of this article.

\section{References}

1 American Psychiatric Association. Diagnostic and Statistical Manual of Mental Disorders (4th edn) (DSM-IV). APA, 1994

2 World Health Organization. The ICD-10 Classification of Mental and Behavioural Disorders: Clinical Descriptions and Diagnostic Guidelines. WHO, 1992.

3 American Psychiatric Association. Diagnostic and Statistical Manual of Mental Disorders (3rd edn) (DSM-IIII). APA, 1980.

4 Charney D, Barlow D, Botteron K, Cohen J, Goldman D, Gur R, et al. Neuroscience research agenda to guide development of a pathophysiologically based classification system. In A Research Agenda for DSM-V (1st edn) (eds DJ Kupfer, MB First, DA Regier): 31-84. American Psychiatric Publishing, 2002.

5 Hyman SE. Neuroscience, genetics, and the future of psychiatric diagnosis Psychopathology 2002; 35: 139-44.

6 Hyman SE. Can neuroscience be integrated into the DSM-V? Nat ReV Neurosci 2007; 8: 725-32.

7 Kendler KS. An historical framework for psychiatric nosology. Psychol Med 2009; 39: 1935-41.

8 American Psychiatric Association. Diagnostic and Statistical Manual of Mental Disorders (3rd edn, revised) (DSM-III-R). APA, 1987.

9 Kuhn TS. The Structure of Scientific Revolutions (3rd edn). University of Chicago Press, 1996

10 Murphy D. Psychiatry in the Scientific Image (Philosophical Psychopathology) (1st edn). MIT Press, 2006.

11 Kupfer DJ, First MB, Regier DA. Introduction. In A Research Agenda for DSM-V (1st edn) (eds DJ Kupfer, MB First, DA Regier): xV-xxiii. American Psychiatric Publishing, 2002.

12 Kendler KS. Reflections on the relationship between psychiatric genetics and psychiatric nosology. Am J Psychiatry 2006; 163: 1138-46.

13 Craddock N, Kendler K, Neale M, Nurnberger J, Purcell S, Rietschel M, et al. Dissecting the phenotype in genome-wide association studies of psychiatric illness. Br J Psychiatry 2009; 195: 97-9.

14 Boyd R. Realism, antifoundationalism and the enthusiasm for natural kinds Philos Stud 1991; 61: 127-48.

15 American Psychiatric Association. Diagnostic and Statistical Manual of Mental Disorders (2nd edn) (DSM-II). APA, 1970.

16 American Psychiatric Association. Diagnostic and Statistical Manual of Mental Disorders (1st edn). APA, 1970.

17 Feighner JP, Robins E, Guze SB, Woodruff Jr RA, Winokur G, Munoz R. Diagnostic criteria for use in psychiatric research. Arch Gen Psychiatry 1972; 26: $57-63$.

18 Spitzer RL, Endicott J, Robins E. Research Diagnostic Criteria for a Selected Group of Functional Disorders (2nd edn). New York Psychiatric Institute, 1975

19 Kupfer DJ, First MB, Regier DA. A Research Agenda for DSM-V (1st edn). American Psychiatric Publishing, 2002.

20 Regier DA, Narrow WE, Kuhl EA, Kupfer DJ. The conceptual development of DSM-V. Am J Psychiatry 2009; 166: 645-50. 